\title{
DYNAMICS OF A NONAUTONOMOUS \\ SEMIRATIO-DEPENDENT PREDATOR-PREY SYSTEM \\ WITH NONMONOTONIC FUNCTIONAL RESPONSES
}

HAI-FENG HUO AND WAN-TONG LI

Received 14 February 2006; Accepted 25 April 2006

A nonautonomous semiratio-dependent predator-prey system with nonmonotonic functional responses is investigated. For general nonautonomous case, positive invariance, permanence, and globally asymptotic stability for the system are studied. For the periodic (almost periodic) case, sufficient conditions for existence, uniqueness, and stability of a positive periodic (almost periodic) solution are obtained.

Copyright (c) 2006 H.-F. Huo and W.-T. Li. This is an open access article distributed under the Creative Commons Attribution License, which permits unrestricted use, distribution, and reproduction in any medium, provided the original work is properly cited.

\section{Introduction}

The Lotka-Volterra system is a rudimentary model on mathematical ecology and has been studied extensively in $[6,8-16,19,24-26]$ and the references cited therein. But in the Lotka-Volterra model, the fact that there are upper limits to the rates of increase of both prey and predator is not recognized. In [17, 18], Leslie introduced a predator-prey model where the "carrying capacity" of the predator's environment is proportional to the number of prey. Leslie stresses the above fact. In the case of continuous time, these considerations lead to the following autonomous model:

$$
\begin{gathered}
\frac{d H}{d t}=\left(r_{1}-b_{1} H\right) H-a_{1} P H, \\
\frac{d P}{d t}=\left(r_{2}-a_{2} \frac{P}{H}\right) P,
\end{gathered}
$$

which is known as the Leslie-Gower predator-prey model [20]. If we assume that the predator consumes the prey according to the functional response $f(H)$, then the system (1.1) formulates as the following:

$$
\frac{d H}{d t}=\left(r_{1}-b_{1} H\right) H-f(H) P, \quad \frac{d P}{d t}=\left(r_{2}-a_{2} \frac{P}{H}\right) P,
$$

Hindawi Publishing Corporation

Discrete Dynamics in Nature and Society

Volume 2006, Article ID 70656, Pages 1-19

DOI 10.1155/DDNS/2006/70656 
which is the so-called semiratio-dependent predator-prey system with the functional responses. Recently, Wang et al. [23] explored the dynamics of a class of the nonautonomous semiratio-dependent predator-prey systems with the functional responses

$$
\begin{gathered}
\frac{d H}{d t}=\left(r_{1}(t)-b_{1}(t) H\right) H-f(t, H) P, \\
\frac{d P}{d t}=\left(r_{2}(t)-a_{2}(t) \frac{P}{H}\right) P,
\end{gathered}
$$

where $H$ and $P$ stand for the population of the prey and the predator, respectively, $f(t, H)$ is the so-called predator functional response to prey, which describes the uptake of substrate by the microorganisms in microbial dynamics or chemical kinetics, and $\partial f(t, H) /$ $\partial H>0$ for $H>0$, which implies that $f(t, H)$ is monotonic function with respect to $H$. However, there are experiments that indicate that nonmonotonic responses occur at the microbial level: when the nutrient concentration reaches a high level, an inhibitory effect on the specific growth rate may occur. This is often seen when microorganisms are used for waste decomposition or for water purification, see Bush and Cook [3]. The so-called Monod-Haldane function

$$
f(H)=\frac{c H}{m^{2}+b H+H^{2}}
$$

has been proposed and used to model the inhibitory effect at high concentrations, see Andrews [1]. Collings [4] also used this response function to model mite predator-prey interactions and called it a Holling IV function. In experiments on the uptake of phenol by pure culture of Pseudomonas putida growing on phenol in continuous culture, Sokol and Howell [22] proposed a simplified Monod-Haldane function of the form

$$
f(H)=\frac{c H}{m^{2}+H^{2}}
$$

and found that it fits their experimental data significantly better than the Monod-Haldane function and is simpler since it involves only two parameters. We would like to call this function a simplified Monod-Haldane or Holling IV response function.

So it is very interesting to study dynamics of a class of the so-called semiratio-dependent predator-prey systems with the nonmonotonic functional responses

$$
\begin{gathered}
\frac{d H(t)}{d t}=\left(r_{1}(t)-\frac{a_{1}(t)}{m^{2}+H^{2}(t)} P(t)-b_{1}(t) H(t)\right) H(t), \\
\frac{d P(t)}{d t}=\left(r_{2}(t)-a_{2}(t) \frac{P(t)}{H(t)}\right) P(t) .
\end{gathered}
$$

For the ecological sense of the system (1.6) we refer to $[5,21,23,27]$ and the references cited therein.

The plan of this paper is as follows: in Section 2, for general case, we will explore some basic problems for (1.6), such as positive invariance, permanence, and globally asymptotic stability for the system (1.6). In Section 3, for periodic case, sufficient conditions for 
existence, uniqueness, and stability of a positive periodic solution are obtained. Section 4 is devoted to the case when all parameters are almost periodic, sufficient conditions for existence, uniqueness and stability of an almost periodic solution are also obtained. The methods used here will be comparison theorems, coincidence degree theory, and Liapunov function.

\section{General nonautonomous case}

In this section, we will address such properties as boundedness of solutions, permanence, and globally asymptotic stability of system (1.6). In the following discussion, we always assume that $r_{i}(t), i=1,2, a_{i}(t), i=1,2$, and $b_{1}(t)$ are all continuous and bounded above and below by positive constants.

Let $\mathbb{R}_{+}^{2}=\left\{(H, P) \in \mathbb{R}^{2} \mid H \geq 0, P \geq 0\right\}$. For a bounded continuous function $g(t)$ on $\mathbb{R}$, we use the following notations:

$$
g^{u}=\sup _{t \in \mathbb{R}} g(t), \quad g^{l}=\inf _{t \in \mathbb{R}} g(t)
$$

LEMMA 2.1. Both the nonnegative and positive cones of $\mathbb{R}^{2}$ are positively invariant for system (1.6).

Proof. Note that system (1.6) is equivalent to

$$
\begin{gathered}
H(t)=H\left(t_{0}\right) \exp \left\{\int_{t_{0}}^{t}\left(r_{1}(s)-\frac{a_{1}(s)}{m^{2}+H^{2}(s)} P(s)-b_{1}(s) H(s)\right) d s\right\}, \\
P(t)=P\left(t_{0}\right) \exp \left\{\int_{t_{0}}^{t}\left(r_{2}(s)-a_{2}(s) \frac{P(s)}{H(s)}\right) d s\right\} .
\end{gathered}
$$

The assertion of the lemma follows immediately for all $t \geq t_{0}$. The proof is complete.

In the remainder of this paper, for biological reasons, we only consider solutions $(H(t)$, $P(t))$ with $H\left(t_{0}\right)>0$ and $P\left(t_{0}\right)>0$.

Definition 2.2. The solution of system (1.6) is said to be ultimately bounded if there exists $B>0$ such that for every solution $(H(t), P(t))$ of system (1.6), there exists $T>0$ such that $\|(H(t), P(t))\| \leq B$, for all $t \geq t_{0}+T$, where $B$ is independent of particular solution while $T$ may depend on the solution.

Definition 2.3. System (1.6) is said to be permanent if there exist positive constants $\delta, \Delta$ with $0<\delta<\Delta$ such that

$$
\begin{aligned}
& \min \left\{\lim _{t \rightarrow+\infty} \inf H(t), \lim _{t \rightarrow+\infty} \inf P(t)\right\} \geq \delta, \\
& \max \left\{\lim _{t \rightarrow+\infty} \sup H(t), \lim _{t \rightarrow+\infty} \sup P(t)\right\} \leq \Delta,
\end{aligned}
$$

for all solutions of system (1.6) with positive initial values. 
4 A semiratio-dependent predator-prey system

Theorem 2.4. If

$$
r_{1}^{l}-\frac{a_{1}^{u}}{m^{2}} M_{2}^{\epsilon}>0
$$

then the set $\Gamma_{\epsilon}$ defined by

$$
\Gamma_{\epsilon}=\left\{(H(t), P(t)) \in \mathbb{R}^{2} \mid m_{1}^{\epsilon} \leq H \leq M_{1}^{\epsilon}, m_{2}^{\epsilon} \leq P \leq M_{2}^{\epsilon}\right\}
$$

is positively invariant with respect to system (1.6), where

$$
\begin{gathered}
M_{1}^{\epsilon}=\frac{r_{1}^{u}}{b_{1}^{l}}+\epsilon, \quad M_{2}^{\epsilon}=\frac{r_{2}^{u} M_{1}^{\epsilon}}{a_{2}^{l}}, \\
m_{1}^{\epsilon}=\frac{r_{1}^{l}-\left(a_{1}^{u} / m^{2}\right) M_{2}^{\epsilon}}{b_{1}^{u}}-\epsilon, \quad m_{2}^{\epsilon}=\frac{r_{2}^{l} m_{1}^{\epsilon}}{a_{2}^{u}},
\end{gathered}
$$

and $\epsilon \geq 0$ is sufficiently small so that $m_{1}^{\epsilon}>0$.

Proof. Let $(H(t), P(t))$ be the solution of system (1.6) through $\left(H\left(t_{0}\right), P\left(t_{0}\right)\right)$ with

$$
m_{1}^{\epsilon} \leq H\left(t_{0}\right) \leq M_{1}^{\epsilon}, \quad m_{2}^{\epsilon} \leq P\left(t_{0}\right) \leq M_{2}^{\epsilon} .
$$

From the first equation in (1.6) and the positivity of the solutions of (1.6), it follows that

$$
\begin{aligned}
H^{\prime}(t) & \leq H(t)\left(r_{1}(t)-b_{1}(t) H(t)\right) \leq H(t)\left(r_{1}^{u}-b_{1}^{l} H(t)\right) \\
& \leq b_{1}^{l} H(t)\left(\frac{r_{1}^{u}}{b_{1}^{l}}+\epsilon-H(t)\right)=b_{1}^{l} H(t)\left(M_{1}^{\epsilon}-H(t)\right), \quad t \geq t_{0} .
\end{aligned}
$$

A standard comparison argument shows that

$$
0<H\left(t_{0}\right) \leq M_{1}^{\epsilon} \Longrightarrow H(t) \leq M_{1}^{\epsilon}, \quad t \geq t_{0}
$$

which together with the second equation in (1.6) produces

$$
\begin{aligned}
P^{\prime}(t) & \leq P(t)\left(r_{2}^{u}-a_{2}^{l} \frac{P(t)}{M_{1}^{\epsilon}}\right)=\frac{a_{2}^{l}}{M_{1}^{\epsilon}} P(t)\left(\frac{r_{2}^{u} M_{1}^{\epsilon}}{a_{2}^{l}}-P(t)\right) \\
& =\frac{a_{2}^{l}}{M_{1}^{\epsilon}} P(t)\left(M_{2}^{\epsilon}-P(t)\right), \quad t \geq t_{0},
\end{aligned}
$$

and hence

$$
0<P\left(t_{0}\right) \leq M_{2}^{\epsilon} \Longrightarrow P(t) \leq M_{2}^{\epsilon}, \quad t \geq t_{0} .
$$


Similarly, the first equation of system (1.6) also yields

$$
\begin{aligned}
H^{\prime}(t) & \geq H(t)\left(r_{1}^{l}-a_{1}^{u} P(t)-b_{1}^{u} H(t)\right) \geq H(t)\left(r_{1}^{l}-\frac{a_{1}^{u}}{m^{2}} M_{2}^{\epsilon}-H(t)\right) \\
& \geq b_{1}^{u} H(t)\left(\frac{r_{1}^{l}-\left(a_{1}^{u} / m^{2}\right) M_{2}^{\epsilon}}{b_{1}^{u}}-\epsilon-H(t)\right)=b_{1}^{u} H(t)\left(m_{1}^{\epsilon}-H(t)\right), \quad t \geq t_{0},
\end{aligned}
$$

and therefore,

$$
H\left(t_{0}\right) \geq m_{1}^{\epsilon} \Longrightarrow H(t) \geq m_{1}^{\epsilon}, \quad t \geq t_{0} .
$$

Moreover, by the second equation of system (1.6), we have

$$
\begin{aligned}
P^{\prime}(t) & \geq P(t)\left(r_{2}^{l}-a_{2}^{u} \frac{P(t)}{m_{1}^{\epsilon}}\right)=\frac{a_{2}^{u}}{m_{1}^{\epsilon}} P(t)\left(\frac{r_{2}^{l} m_{1}^{\epsilon}}{a_{2}^{u}}-P(t)\right) \\
& =\frac{a_{2}^{u}}{m_{1}^{\epsilon}} P(t)\left(m_{2}^{\epsilon}-P(t)\right), \quad t \geq t_{0},
\end{aligned}
$$

which implies

$$
P\left(t_{0}\right) \geq m_{2}^{\epsilon} \Longrightarrow P(t) \geq m_{2}^{\epsilon}, \quad t \geq t_{0} .
$$

Thus, $\Gamma_{\epsilon}$ is positive invariant for system (1.6), and the proof is complete.

Lemma 2.5. Let $(H(t), P(t))$ be a solution of system (1.6) with $H\left(t_{0}\right)>0$ and $P\left(t_{0}\right)>0$. Then,

$$
\limsup _{t \rightarrow \infty} H(t) \leq M_{1}^{0}
$$

Moreover, assume that (2.4) holds, then

$$
\liminf _{t \rightarrow \infty} H(t) \geq m_{1}^{0} .
$$

Proof. Noting that (2.8) and (2.12) are valid, the conclusion follows from a standard comparison arguments directly.

For the predator population, we can also have some estimates.

Lemma 2.6. Assume that (2.4) holds, then

$$
\liminf _{t \rightarrow \infty} P(t) \geq m_{2}^{0}, \quad \limsup _{t \rightarrow \infty} P(t) \leq M_{2}^{0} .
$$

Proof. Since $\limsup \sup _{t \rightarrow \infty} H(t) \leq M_{1}^{0}$, for any sufficient small $\epsilon>0$, there is some $t_{1}>t_{0}$ such that for $t \geq t_{1}$,

$$
H(t) \leq M_{1}^{0}+\epsilon
$$


Then, from the second equation of system (1.6), it follows that

$$
\begin{aligned}
P^{\prime}(t) & \leq P(t)\left(r_{2}^{u}-a_{2}^{l} \frac{P(t)}{M_{1}^{\epsilon}}\right)=\frac{a_{2}^{l}}{M_{1}^{\epsilon}} P(t)\left(\frac{r_{2}^{u} M_{1}^{\epsilon}}{a_{2}^{l}}-P(t)\right) \\
& =\frac{a_{2}^{l}}{M_{1}^{\epsilon}} P(t)\left(M_{2}^{\epsilon}-P(t)\right), \quad t \geq t_{1} .
\end{aligned}
$$

Hence, by using the comparison theorem of ordinary differential equations and the arbitrariness of $\epsilon$, we have

$$
\limsup _{t \rightarrow \infty} P(t) \leq M_{2}^{0}
$$

By a similar argument, we can easily show that

$$
\liminf _{t \rightarrow \infty} P(t) \geq m_{2}^{0}
$$

The proof is complete.

Lemmas 2.5 and 2.6 immediately lead to the following.

Theorem 2.7. Assume that (2.4) holds, then system (1.6) is permanent.

From the proofs of Lemmas 2.5 and 2.6, we can easily obtain the following ultimate boundedness of $\Gamma_{\epsilon}$ with $\epsilon>0$ sufficiently small.

Theorem 2.8. Assume that (2.4) holds, then the set $\Gamma_{\epsilon}$ with $\epsilon>0$ defined by (2.5) is an ultimately bounded region of system (1.6).

Definition 2.9. A bounded nonnegative solution $\left(H^{*}(t), P^{*}(t)\right)$ of $(1.6)$ is said to be globally asymptotically stable (or globally attractive) if for any other solution $(H(t), P(t))$ of (1.6) with positive initial values the following holds:

$$
\lim _{t \rightarrow+\infty}\left(\left|H(t)-H^{*}(t)\right|+\left|P(t)-P^{*}(t)\right|\right)=0
$$

Remark 2.10. In general, if the above property holds for any two solutions with positive initial values, then we say system (1.6) is globally asymptotically stable. One can easily show that if system (1.6) has a bounded positive solution which is globally asymptotically stable, then system (1.6) is globally asymptotically stable, and vice versa.

The following lemma is from Barbalat [2], and will be employed in establishing the globally asymptotic stability of system (1.6).

Lемма 2.11. let $h$ be a real number and let $f$ be a nonnegative function defined on $[h,+\infty)$ such that $f$ is integrable on $[h,+\infty)$ and is uniformly continuous on $[h,+\infty)$, then $\lim _{t \rightarrow+\infty} f(t)=0$. 
Theorem 2.12. Let $\left(H^{*}(t), P^{*}(t)\right)$ be a bounded positive solution of system (1.6). Assume that (2.4) holds, and

$$
\begin{gathered}
b_{1}^{l}(t)+\frac{2 m_{2}^{\epsilon} m_{1}^{\epsilon}}{\left(m^{2}+\left(M_{1}^{\epsilon}\right)^{2}\right)^{2}}-\frac{M_{2}^{\epsilon}}{\left(m_{1}^{\epsilon}\right)^{2}}>0, \\
\frac{1}{M_{1}^{\epsilon}}-a_{1}^{u}(t)-\frac{1}{\left(m^{2}+\left(m_{1}^{\epsilon}\right)^{2}\right)}>0,
\end{gathered}
$$

where $M_{i}^{\epsilon}, m_{i}^{\epsilon}, i=1,2$, are defined in (2.6). Then $\left(H^{*}(t), P^{*}(t)\right)$ is globally asymptotically stable.

Proof. Let $(H(t), P(t))$ be any solution of system (1.6) with a positive initial value. Since $\Gamma_{\epsilon}$ is an ultimately bounded region of system (1.6), there exists a $T_{1}>0$, such that $(H(t)$, $P(t)) \in \Gamma_{\epsilon}$ and $\left(H^{*}(t), P^{*}(t)\right) \in \Gamma_{\epsilon}$ for all $t \geq t_{0}+T_{1}$.

Consider a Lyapunov function defined by

$$
V(t)=\left|\ln \{H(t)\}-\ln \left\{H^{*}(t)\right\}\right|+\left|\ln \{P(t)\}-\ln \left\{P^{*}(t)\right\}\right|, \quad t \geq t_{0} .
$$

Calculating the right derivative of $V(t)$ along the solution of system (1.6), we derive for $t \geq t_{0}+T_{1}$ that

$$
\begin{aligned}
D^{+} V(t)= & \operatorname{sgn}\left(H(t)-H^{*}(t)\right) \\
& \times\left(-a_{1}(t)\left(\frac{P(t)}{m^{2}+H^{2}(t)}-\frac{P^{*}(t)}{m^{2}+\left(H^{*}(t)\right)^{2}}\right)-b_{1}(t)\left(H(t)-H^{*}(t)\right)\right) \\
& +\operatorname{sgn}\left(P(t)-P^{*}(t)\right)\left(-a_{2}(t)\left(\frac{P(t)}{H(t)}-\frac{P^{*}(t)}{H^{*}(t)}\right)\right) \\
\leq & -\left(b_{1}^{l}+\frac{P^{*}(t)\left(H(t)+H^{*}(t)\right)}{\left(m^{2}+H^{2}(t)\right)\left(m^{2}+\left(H^{*}(t)\right)^{2}\right)}-\frac{P(t)}{H(t) H^{*}(t)}\right)\left|H(t)-H^{*}(t)\right| \\
& -\left(\frac{1}{H^{*}(t)}-a_{1}^{u}-\frac{1}{\left(m^{2}+H^{2}(t)\right)}\right)\left|P(t)-P^{*}(t)\right| \\
\leq & -\left(b_{1}^{l}+\frac{2 m_{2}^{\epsilon} m_{1}^{\epsilon}}{\left(m^{2}+\left(M_{1}^{\epsilon}\right)^{2}\right)^{2}}-\frac{M_{2}^{\epsilon}}{\left(m_{1}^{\epsilon}\right)^{2}}\right)\left|H(t)-H^{*}(t)\right| \\
& -\left(\frac{1}{M_{1}^{\epsilon}}-a_{1}^{u}-\frac{1}{\left(m^{2}+\left(m_{1}^{\epsilon}\right)^{2}\right)}\right)\left|P(t)-P^{*}(t)\right| .
\end{aligned}
$$

From (2.24) it follows that there exists a positive constant $\mu>0$ such that

$$
D^{+} V(t) \leq-\mu\left[\left|H(t)-H^{*}(t)\right|+\left|P(t)-P^{*}(t)\right|\right], \quad t \geq t_{0}+T_{1} .
$$


Integrating on both sides of (2.27) from $t_{0}+T_{1}$ to $t$ produces

$$
\begin{aligned}
V(t) & +\mu \int_{t_{0}+T_{1}}^{t}\left[\left|H(s)-H^{*}(s)\right|+\left|P(s)-P^{*}(s)\right|\right] d s \\
& \leq V\left(t_{0}+T_{1}\right)<+\infty, \quad t \geq t_{0}+T_{1} .
\end{aligned}
$$

Then

$$
\int_{t_{0}+T_{1}}^{t}\left[\left|H(s)-H^{*}(s)\right|+\left|P(s)-P^{*}(s)\right|\right] d s \leq \mu^{-1} V\left(t_{0}+T_{1}\right)<+\infty, \quad t \geq t_{0}+T_{1},
$$

and hence, $\left|H(t)-H^{*}(t)\right|+\left|P(t)-P^{*}(t)\right| \in L^{1}\left(\left[t_{0}+T_{1},+\infty\right)\right)$.

The boundedness of $H^{*}(t)$ and $P^{*}(t)$ and the ultimate boundedness of $H(t)$ and $P(t)$ imply that $H(t), P(t), H^{*}(t)$, and $P^{*}(t)$ all have a bounded derivative for $t \geq t_{0}+T_{1}$. Then, it follows that $\left|H(t)-H^{*}(t)\right|+\left|P(t)-P^{*}(t)\right|$ is uniformly continuous on $\left[t_{0}+\right.$ $\left.T_{1},+\infty\right)$. By Lemma 2.11, we have

$$
\lim _{t \rightarrow+\infty}\left(\left|H(t)-H^{*}(t)\right|+\left|P(t)-P^{*}(t)\right|\right)=0 .
$$

The proof is complete.

\section{Periodic case}

Throughout this section, we will assume that $r_{i}(t), a_{i}(t), i=1,2, b_{1}(t)$, are positive $\omega$ period functions, that is, system (1.6) is $\omega$-period system and will study the existence and stability of a positive periodic solution of (1.6).

LemMa 3.1 (Brouwer fixed point theorem). Let $\sigma$ be a continuous operator that maps a closed, bounded, convex subset $\bar{\Omega} \subset \mathbb{R}^{n}$ into itself. Then $\bar{\Omega}$ contains at least one fixed point of the operator $\sigma$, that is, there exists an $x^{*} \in \bar{\Omega}$ such that $\sigma\left(x^{*}\right)=x^{*}$.

Theorem 3.2. Assume that (2.4) holds, then system (1.6) has at least one positive periodic solution of period $\omega$, say $(\bar{H}(t), \bar{P}(t))$ which lies in $\Gamma_{\epsilon}$.

Proof. Define a shift operator, which is also known as a Poincaré mapping $\sigma: \mathbb{R}^{2} \rightarrow \mathbb{R}^{2}$ by

$$
\sigma\left(\left(H_{0}, P_{0}\right)\right)=\left(H\left(\omega, t_{0},\left(H_{0}, P_{0}\right)\right), P\left(\omega, t_{0},\left(H_{0}, P_{0}\right)\right)\right), \quad\left(H_{0}, P_{0}\right) \in \mathbb{R}^{2},
$$

where $\left(H\left(\omega, t_{0},\left(H_{0}, P_{0}\right)\right), P\left(\omega, t_{0},\left(H_{0}, P_{0}\right)\right)\right)$ denotes the solution of system (1.6) through the point $\left(t_{0},\left(H_{0}, P_{0}\right)\right)$. Theorem 2.4 tells us that $\Gamma_{\epsilon}$ is a positive invariant with respect to system (1.6), and hence, the operator $\sigma$ defined above maps $\Gamma_{\epsilon}$ into itself, that is, $\sigma\left(\Gamma_{\epsilon}\right) \subset$ $\Gamma_{\epsilon}$. Since the solution of system (1.6) is continuous with respect to the initial value, the operator $\sigma$ is continuous. It is easy to show that $\Gamma_{\epsilon}$ is a bounded, closed, convex set in $\mathbb{R}^{2}$. By Lemma 3.1, $\sigma$ has at least one fixed point in $\Gamma_{\epsilon}$, that is, there exists an $(\bar{H}(t), \bar{P}(t)) \in \Gamma_{\epsilon}$ such that

$$
(\bar{H}(t), \bar{P}(t))=\left(H\left(\omega, t_{0},(\bar{H}, \bar{P})\right), P\left(\omega, t_{0},(\bar{H}, \bar{P})\right)\right) .
$$


Therefore, there exists at least one positive periodic solution, say $(\bar{H}(t), \bar{P}(t))$, and the invariance of $\Gamma_{\epsilon}$ assures that $(\bar{H}(t), \bar{P}(t)) \in \Gamma_{\epsilon}$. The proof is complete.

Next, we will employ an alternative approach, that is, a continuation theorem in coincidence degree theorem, to establish some different criteria for the same problem. To this end, we need some preparation as follows.

Let $X$ and $Z$ be two Banach spaces. Consider an operator equation

$$
L x=\lambda N x, \quad \lambda \in(0,1)
$$

where $L: \operatorname{Dom} L \cap X \rightarrow Z$ is a linear operator and $\lambda$ is a parameter. Let $P$ and $Q$ denote two projectors such that

$$
P: X \cap \operatorname{Dom} L \longrightarrow \operatorname{Ker} L, \quad Q: Z \longrightarrow Z / \operatorname{Im} L
$$

In the sequel, we will use the following result of Gaines and Mawhin [7, page 40].

Lemma 3.3. Let $X$ and $Z$ be two Banach spaces and let $L$ be a Fredholm mapping of index zero. Assume that $N: \bar{\Omega} \rightarrow Z$ is L-compact on $\bar{\Omega}$ with $\Omega$ open bounded in X. Furthermore assume that

(a) for each $\lambda \in(0,1), x \in \partial \Omega \cap \operatorname{Dom} L$,

$$
L x \neq \lambda N x
$$

(b) for each $x \in \partial \Omega \cap \operatorname{Ker} L$,

$$
Q N x \neq 0, \quad \operatorname{deg}\{Q N x, \Omega \cap \operatorname{Ker} L, 0\} \neq 0 .
$$

Then the equation $L x=N x$ has at least one solution in $\bar{\Omega} \cap \operatorname{Dom} L$.

Recall that a linear mapping $L: \operatorname{Dom} L \cap X \rightarrow Z$ with $\operatorname{Ker} L=L^{-1}(0)$ and $\operatorname{Im} L=$ $L(\operatorname{Dom} L)$ will be called a Fredholm mapping if the following two conditions hold:

(i) $\operatorname{Ker} L$ has a finite dimension;

(ii) $\operatorname{Im} L$ is closed and has a finite codimension.

Recall also that the codimension of $\operatorname{Im} L$ is the dimension of $Z / \operatorname{Im} L$, that is, the dimension of the cokernel coker $L$ of $L$.

When $L$ is a Fredholm mapping, its index is the integer $\operatorname{Ind} L=\operatorname{dim} \operatorname{ker} L-\operatorname{codim} \operatorname{Im} L$.

We will say that a mapping $N$ is $L$-compact on $\Omega$ if the mapping $Q N: \bar{\Omega} \rightarrow Z$ is continuous, $Q N(\bar{\Omega})$ is bounded, and $K_{p}(I-Q) N: \bar{\Omega} \rightarrow X$ is compact, that is, it is continuous and $K_{p}(I-Q) N(\bar{\Omega})$ is relatively compact, where $K_{p}: \operatorname{Im} L \rightarrow \operatorname{Dom} L \cap \operatorname{Ker} P$ is a inverse of the restriction $L_{p}$ of $L$ to $\operatorname{Dom} L \cap \operatorname{Ker} P$, so that $L K_{p}=I$ and $K_{p} L=I-P$.

For convenience, we will introduce the notation

$$
\bar{u}=\frac{1}{\omega} \int_{0}^{\omega} u(t) d t
$$

where $u$ is a periodic continuous function with period $\omega$. 
THEOREM 3.4. If the system of algebraic equations

$$
\begin{gathered}
\bar{r}_{1}-\frac{\bar{a}_{1}}{m^{2}+v_{1}^{2}} v_{2}-\bar{b}_{1} v_{1}=0, \\
\bar{r}_{2}-\bar{a}_{2} \frac{v_{2}}{v_{1}}=0
\end{gathered}
$$

has a unique solution $\left(v_{1}^{*}, v_{2}^{*}\right)^{T} \in \operatorname{int} \mathbb{R}_{+}^{2}$ with $v_{i}^{*}>0, i=1,2$, then system (1.6) has at least one positive $\omega$-periodic solution.

Proof. Since

$$
\begin{gathered}
H(t)=H(0) \exp \left\{\int_{0}^{t}\left[r_{1}(s)-\frac{a_{1}(s)}{m^{2}+H^{2}(s)} P(s)-b_{1}(s) H(s)\right] d s\right\}, \\
P(t)=P(0) \exp \left\{\int_{0}^{t}\left[r_{2}(s)-a_{2}(s) \frac{P(s)}{H(s)}\right] d s\right\},
\end{gathered}
$$

the solution of system (1.6) remains positive for $t \geq 0$, we can let

$$
H(t)=\exp \left\{x_{1}(t)\right\}, \quad P(t)=\exp \left\{x_{2}(t)\right\}
$$

and derive that

$$
\begin{gathered}
\frac{d x_{1}(t)}{d t}=r_{1}(t)-\frac{a_{1}(t)}{m^{2}+\exp \left[2 x_{1}(t)\right]} \exp \left[x_{2}(t)\right]-b_{1}(t) \exp \left[x_{1}(t)\right], \\
\frac{d x_{2}(t)}{d t}=r_{2}(t)-a_{2}(t) \frac{\exp \left\{x_{2}(t)\right\}}{\exp \left\{x_{1}(t)\right\}} .
\end{gathered}
$$

In order to use Lemma 2.1 to system (1.6), we take

$$
X=Z=\left\{x(t)=\left(x_{1}(t), x_{2}(t)\right)^{T} \in C\left(\mathbb{R}, \mathbb{R}^{2}\right): x(t+\omega)=x(t)\right\}
$$

and denote

$$
\|x\|=\left\|\left(x_{1}(t), x_{2}(t)\right)^{T}\right\|=\max _{t \in[0, \omega]}\left|x_{1}(t)\right|+\max _{t \in[0, \omega]}\left|x_{2}(t)\right| .
$$

Then $X$ and $Z$ are Banach spaces when they are endowed with the norms $\|\cdot\|$.

Set

$$
\begin{gathered}
N x=\left[\begin{array}{c}
r_{1}(t)-\frac{a_{1}(t)}{m^{2}+\exp \left[2 x_{1}(t)\right]} \exp \left[x_{2}(t)\right]-b_{1}(t) \exp \left[x_{1}(t)\right] \\
r_{2}(t)-a_{2}(t) \frac{\exp \left\{x_{2}(t)\right\}}{\exp \left[x_{1}(t)\right]}
\end{array}\right], \\
L x=x^{\prime}, \quad P x=\frac{1}{\omega} \int_{0}^{\omega} x(t) d t, \quad x \in X, \quad Q z=\frac{1}{\omega} \int_{0}^{\omega} z(t) d t, \quad z \in \mathbb{Z} .
\end{gathered}
$$

Evidently, $\operatorname{Ker} L=\left\{x \mid x \in X, x=\mathbb{R}^{2}\right\}, \operatorname{Im} L=\left\{z \mid z \in Z, \int_{0}^{\omega} z(t) d t=0\right\}$ is closed in $\mathbb{Z}$ and $\operatorname{dim} \operatorname{Ker} L=\operatorname{codim} \operatorname{Im} L=2$. Hence, $L$ is a Fredholm mapping of index zero. Furthermore, 
the generalized inverse (to $L) K_{p}: \operatorname{Im} L \rightarrow \operatorname{Ker} P \cap \operatorname{dom} L$ has the form

$$
K_{p}(z)=\int_{0}^{t} z(s) d s-\frac{1}{\omega} \int_{0}^{\omega} \int_{0}^{t} z(s) d s d t
$$

Thus

$$
Q N x=\left[\begin{array}{c}
\frac{1}{\omega} \int_{0}^{\omega}\left[r_{1}(t)-\frac{a_{1}(t)}{m^{2}+\exp \left[2 x_{1}(t)\right]} \exp \left\{x_{2}(t)\right\}-b_{1}(t) \exp \left\{x_{1}(t)\right\}\right] d t \\
\frac{1}{\omega} \int_{0}^{\omega}\left[r_{2}(t)-a_{2}(t) \frac{\exp \left\{x_{2}(t)\right\}}{\exp \left\{x_{1}(t)\right\}}\right] d t
\end{array}\right],
$$

$K_{p}(I-Q) N$

$$
\begin{aligned}
& =\left[\begin{array}{c}
\int_{0}^{t}\left[r_{1}(s)-\frac{a_{1}(s)}{m^{2}+\exp \left[2 x_{1}(s)\right]} \exp \left\{x_{2}(s)\right\}-b_{1}(s) \exp \left\{x_{1}(s)\right\}\right] d s \\
\int_{0}^{t}\left[r_{2}(s)-a_{2}(s) \frac{\exp \left\{x_{2}(s)\right\}}{\exp \left\{x_{1}(s)\right\}}\right] d s
\end{array}\right] \\
& -\left[\begin{array}{c}
\frac{1}{\omega} \int_{0}^{\omega} \int_{0}^{t}\left[r_{1}(s)-\frac{a_{1}(s)}{m^{2}+\exp \left[2 x_{1}(s)\right]} \exp \left\{x_{2}(s)\right\}-b_{1}(s) \exp \left\{x_{1}(s)\right\}\right] d s d t \\
\frac{1}{\omega} \int_{0}^{\omega} \int_{0}^{t}\left[r_{2}(s)-a_{2}(s) \frac{\exp \left\{x_{2}(s)\right\}}{\exp \left\{x_{1}(s)\right\}}\right] d s d t
\end{array}\right] \\
& -\left[\begin{array}{c}
\left(\frac{t}{\omega}-\frac{1}{2}\right) \int_{0}^{\omega}\left[r_{1}(t)-\frac{a_{1}(t)}{m^{2}+\exp \left[2 x_{1}(t)\right]} \exp \left\{x_{2}(t)\right\}-b_{1}(t) \exp \left\{x_{1}(t)\right\}\right] d t \\
\left(\frac{t}{\omega}-\frac{1}{2}\right) \int_{0}^{\omega}\left[r_{2}(t)-a_{2}(t) \frac{\exp \left\{x_{2}(t)\right\}}{\exp \left\{x_{1}(t)\right\}}\right] d t
\end{array}\right] .
\end{aligned}
$$

Clearly, $Q N$ and $K_{p}(I-Q) N$ are continuous and, moreover, $Q N(\bar{\Omega}), K_{p}(I-Q) N(\bar{\Omega})$ are relatively compact for any open bounded set $\Omega \subset X$. Hence, $N$ is $L$-compact on $\bar{\Omega}$, here $\Omega$ is any open bounded set in $X$.

Now we reach the position to search for an appropriate open bounded subset $\Omega$ for the application of Lemma 2.1. Corresponding to equation $L x=\lambda N x, \lambda \in(0,1)$, we have

$$
\begin{gathered}
x_{1}^{\prime}(t)=\lambda\left[r_{1}(t)-\frac{a_{1}(t)}{m^{2}+\exp \left[2 x_{1}(t)\right]} \exp \left\{x_{2}(t)\right\}-b_{1}(t) \exp \left\{x_{1}(t)\right\}\right], \\
x_{2}^{\prime}(t)=\lambda\left[r_{2}(t)-a_{2}(t) \frac{\exp \left\{x_{2}(t)\right\}}{\exp \left\{x_{1}(t)\right\}}\right] .
\end{gathered}
$$


Suppose that $x(t)=\left(x_{1}, x_{2}\right) \in X$ is a solution of system (3.17) for a certain $\lambda \in(0,1)$. By integrating (3.17) over the interval $[0, \omega]$, we obtain

$$
\begin{gathered}
\int_{0}^{\omega}\left[r_{1}(t)-\frac{a_{1}(t)}{m^{2}+\exp \left[2 x_{1}(t)\right]} \exp \left\{x_{2}(t)\right\}-b_{1}(t) \exp \left\{x_{1}(t)\right\}\right] d t=0, \\
\int_{0}^{\omega}\left[r_{2}(t)-a_{2}(t) \frac{\exp \left\{x_{2}(t)\right\}}{\exp \left\{x_{1}(t)\right\}}\right] d t=0 .
\end{gathered}
$$

Hence

$$
\begin{gathered}
\int_{0}^{\omega}\left[\frac{a_{1}(t)}{m^{2}+\exp \left[2 x_{1}(t)\right]} \exp \left\{x_{2}(t)\right\}+b_{1}(t) \exp \left\{x_{1}(t)\right\}\right] d t=\bar{r}_{1} \omega, \\
\int_{0}^{\omega}\left[a_{2}(t) \frac{\exp \left\{x_{2}(t)\right\}}{\exp \left\{x_{1}(t)\right\}}\right] d t=\bar{r}_{2} \omega .
\end{gathered}
$$

From (3.17), (3.19), and (3.20), we obtain

$$
\begin{aligned}
& \int_{0}^{\omega}\left|x_{1}^{\prime}(t)\right| d t< \int_{0}^{\omega}\left[\frac{a_{1}(t)}{m^{2}+\exp \left[2 x_{1}(t)\right]} \exp \left\{x_{2}(t)\right\}+b_{1}(t) \exp \left\{x_{1}(t)\right\}\right] d t \\
&+\int_{0}^{\omega}\left|r_{1}(t)\right| d t=2 \bar{r}_{1} \omega, \\
& \int_{0}^{\omega}\left|x_{2}^{\prime}(t)\right| d t<\int_{0}^{\omega}\left[a_{2}(t) \frac{\exp \left\{x_{2}(t)\right\}}{\exp \left\{x_{1}(t)\right\}}\right] d t+\bar{r}_{2} \omega=2 \bar{r}_{2} \omega .
\end{aligned}
$$

Note that $\left(x_{1}(t), x_{2}(t)\right)^{T} \in X$, then there exists $\xi_{i}, \eta_{i} \in[0, \omega], i=1,2$ such that

$$
x_{i}\left(\xi_{i}\right)=\min _{t \in[0, \omega]} x_{i}(t), \quad x_{i}\left(\eta_{i}\right)=\max _{t \in[0, \omega]} x_{i}(t), \quad i=1,2
$$

By (3.19) and (3.22), we obtain

$$
\begin{aligned}
& \bar{r}_{1} \omega \geq \bar{b}_{1} \omega \exp \left\{x_{1}\left(\xi_{1}\right)\right\}, \quad x_{1}\left(\xi_{1}\right) \leq \ln \left\{\frac{\bar{r}_{1}}{\bar{b}_{1}}\right\}, \\
& x_{1}(t) \leq x_{1}\left(\xi_{1}\right)+\int_{0}^{\omega}\left|x_{1}^{\prime}(t)\right| d t<\ln \left\{\frac{\bar{r}_{1}}{\bar{b}_{1}}\right\}+2 \bar{r}_{1} \omega .
\end{aligned}
$$

In addition, from (3.22) and system (3.17), we obtain

$$
\begin{gathered}
r_{2}\left(\xi_{2}\right)-a_{2}\left(\xi_{2}\right) \frac{\exp \left\{x_{2}\left(\xi_{2}\right)\right\}}{\exp \left\{x_{1}\left(\xi_{2}\right)\right\}}=0, \\
r_{2}\left(\xi_{2}\right)-a_{2}\left(\xi_{2}\right) \frac{1}{\exp \left\{x_{1}\left(\eta_{1}\right)\right\}} \geq 0, \\
x_{1}\left(\eta_{1}\right) \geq \ln \frac{a_{2}\left(\xi_{2}\right)}{r_{2}\left(\xi_{2}\right)} \geq \min _{t \in[0, \infty]}\left\{\ln \left(\frac{a_{2}(t)}{r_{2}(t)}\right)\right\} .
\end{gathered}
$$


Then

$$
x_{1}(t) \geq x_{1}\left(\eta_{1}\right)-\int_{0}^{\omega}\left|x_{1}^{\prime}(t)\right| d t \geq \min _{t \in[0, \infty]}\left\{\ln \left(\frac{a_{2}(t)}{r_{2}(t)}\right)\right\}-2 \bar{r}_{1} \omega .
$$

It follows from (3.24) and (3.26) that

$$
\max _{t \in[0, \omega]}\left|x_{1}(t)\right| \leq \max \left\{\left|\ln \left\{\frac{\bar{r}_{1}}{\bar{b}_{1}}\right\}+2 \bar{r}_{1} \omega\right|,\left|\min _{t \in[0, \infty]}\left\{\ln \left(\frac{a_{2}(t)}{r_{2}(t)}\right)\right\}-2 \bar{r}_{1} \omega\right|\right\}:=M_{1} .
$$

In view of (3.20) and (3.22), we have

$$
\bar{r}_{2} \omega \leq \bar{a}_{2} \omega \exp \left\{x_{2}\left(\eta_{2}\right)\right\}
$$

that is

$$
x_{2}\left(\eta_{2}\right) \geq \ln \left\{\frac{\bar{r}_{2}}{\bar{a}_{2}}\right\}
$$

Then

$$
x_{2}(t) \geq x_{2}\left(\eta_{2}\right)-\int_{0}^{\omega}\left|x_{2}^{\prime}(t)\right| d t<\ln \left\{\frac{\bar{r}_{2}}{\bar{a}_{2}}\right\}-2 \bar{r}_{2} \omega
$$

By virtue of (3.19), (3.24), and (3.22), we obtain that

$$
\bar{r}_{1} \omega \geq \frac{\bar{a}_{1}}{m^{2}+\exp \left[2 M_{1}\right]} \omega \exp \left\{x_{2}\left(\xi_{2}\right)\right\},
$$

and so

$$
x_{2}\left(\xi_{2}\right) \leq \ln \left\{\frac{\bar{r}_{1}\left(m^{2}+\exp \left[2 M_{1}\right]\right)}{\bar{a}_{1}}\right\} \text {. }
$$

Then

$$
x_{2}(t) \leq x_{2}\left(\xi_{2}\right)+\int_{0}^{\omega}\left|x_{2}^{\prime}(t)\right| d t<\ln \left\{\frac{\bar{r}_{1}\left(m^{2}+\exp \left[2 M_{1}\right]\right)}{\bar{a}_{1}}\right\}+2 \bar{r}_{2} \omega .
$$

It follows from (3.30) and (3.33) that

$$
\max _{t \in[0, \omega]}\left|x_{2}(t)\right| \leq \max \left\{\left|\ln \left\{\frac{\bar{r}_{2}}{\bar{a}_{2}}\right\}-2 \bar{r}_{2} \omega\right|,\left|\frac{\bar{r}_{1}\left(m^{2}+\exp \left[2 M_{1}\right]\right)}{\bar{a}_{1}}+2 \bar{r}_{2} \omega\right|\right\}:=M_{2} .
$$

Clearly, $M_{i}, i=1,2$, are independent of $\lambda$. Under the assumption in Theorem 3.4, it is easy to show that the system of algebraic equations

$$
\bar{r}_{1}-\frac{\bar{a}_{1}}{m^{2}+v_{1}^{2}} v_{2}-\bar{b}_{1} v_{1}=0, \quad \bar{r}_{2}-\bar{a}_{2} \frac{v_{2}}{v_{1}}=0
$$


has a unique solution $\left(v_{1}^{*}, v_{2}^{*}\right)^{T} \in \operatorname{int} \mathbb{R}_{+}^{2}$ with $v_{i}^{*}>0, i=1,2$. Denote $M=M_{1}+M_{2}+M_{3}$, where $M_{3}>0$ is taken sufficiently large such that

$$
\left\|\left(\ln \left\{v_{1}^{*}\right\}, \ln \left\{v_{2}^{*}\right\}\right)\right\|=\left|\ln \left\{v_{1}^{*}\right\}\right|+\left|\ln \left\{v_{2}^{*}\right\}\right|<M_{3},
$$

and define

$$
\Omega=\{x(t) \in X:\|x\|<M\} .
$$

It is clear that $\Omega$ satisfies the condition (a) of Lemma 2.1. When

$$
x=\left(x_{1}, x_{2}\right)^{T} \in \partial \Omega \cap \operatorname{Ker} L=\partial \Omega \cap \mathbb{R}^{2},
$$

$x$ is a constant vector in $\mathbb{R}^{2}$ with $\|x\|=M$. Then

$$
Q N x=\left[\begin{array}{c}
\bar{r}_{1}-\frac{\bar{a}_{1}}{m^{2}+v_{1}^{2}} \exp \left\{x_{2}\right\}-\bar{b}_{1} \exp \left\{x_{1}\right\} \\
\bar{r}_{2}-\bar{a}_{2} \frac{\exp \left\{x_{2}\right\}}{\exp \left\{x_{1}\right\}}
\end{array}\right] \neq 0 .
$$

Furthermore, in view of assumption in Theorem 3.4, it can be easily seen that

$$
\operatorname{deg}\{Q N x, \Omega \cap \operatorname{Ker} L, 0\} \neq 0 .
$$

By now we know that $\Omega$ verifies all the requirements of Lemma 3.3 and then system (3.11) has at least one $\omega$-periodic solution. By the medium of (3.10), we derive that system (1.6) has at least one positive $\omega$-periodic solution. The proof is complete.

\section{Almost periodic case}

The assumption of almost periodicity of system (1.6) is a way of incorporating the time dependent variability of the environment, especially when the various components of the environment are periodic with not necessary commensurate period. Mathematically, system (1.6) will denote a generation of an autonomous and periodic system. Therefore, throughout this section, in addition to the assumptions in Section 2, we further assume that $r_{i}(t), a_{i}(t), i=1,2, b_{1}(t)$ are almost periodic. Thus, all the theorems in Section 2 remain valid.

Let

$$
H(t)=\exp (\tilde{H}(t)), \quad P(t)=\exp (\tilde{P}(t)),
$$

then system (1.6) becomes

$$
\begin{gathered}
\frac{d \tilde{H}(t)}{d t}=r_{1}(t)-\frac{a_{1}(t)}{m^{2}+\exp 2(\tilde{H}(t))} \exp (\widetilde{P}(t))-b_{1}(t) \exp (\tilde{H}(t)), \\
\frac{d \tilde{P}(t)}{d t}=r_{2}(t)-a_{2}(t) \frac{\exp \{\widetilde{P}(t)\}}{\exp \{\tilde{H}(t)\}}
\end{gathered}
$$


By Theorem 2.8, it is not difficult to prove the following theorem.

Theorem 4.1. Assume that (2.4) holds, then the set $\Gamma_{\epsilon}$ defined by

$$
\Gamma_{\epsilon}^{*}=\left\{(H(t), P(t)) \in \mathbb{R}^{2} \mid \ln \left(m_{1}^{\epsilon}\right) \leq H \leq \ln \left(M_{1}^{\epsilon}\right), \ln \left(m_{2}^{\epsilon}\right) \leq P \leq \ln \left(M_{2}^{\epsilon}\right)\right\}
$$

is positively invariant and ultimately bounded region of system (4.2), where $M_{i}^{\epsilon}, m_{i}^{\epsilon}, i=1,2$, are defined in (2.6).

In order to prove the main result of this section, we will first introduce a useful lemma. Consider the ordinary differential equation

$$
x^{\prime}=f(t, x), \quad f(t, x) \in C\left(R \times D, \mathbb{R}^{n}\right)
$$

where $D$ is an open set in $\mathbb{R}^{n}, f(t, x)$ is almost periodic in $t$ uniformly with respect to $x \in D$.

To discuss the existence of an almost periodic solution of (4.4), we consider the product system of (4.4)

$$
\begin{aligned}
& x^{\prime}=f(t, x), \\
& y^{\prime}=f(t, y) .
\end{aligned}
$$

Lemma 4.2 [28, Theorem 19.1]. Suppose that there exists a Lyapunov function $V(t, x, y)$ defined on $[0,+\infty) \times D \times D$ which satisfies the following conditions:

(i) $\alpha(\|x-y\|) \leq V(t, x, y) \leq \beta(\|x-y\|)$, where $\alpha(\gamma)$ and $\beta(\gamma)$ are continuous, increasing and positive definite;

(ii) $\left|V\left(t, x_{1}, y_{1}\right)-V\left(t, x_{2}, y_{2}\right)\right| \leq K\left\{\left\|x_{1}-x_{2}\right\|+\left\|y_{1}-y_{2}\right\|\right\}$, where $K>0$ is a constant;

(iii) $V_{(4.3)}^{\prime}(t, x, y) \leq-\mu V(|x-y|)$, where $\mu>0$ is a constant.

Moreover, suppose that system (4.4) has a solution that remains in a compact set $S \subset D$ for all $t \geq t_{0} \geq 0$. Then system (4.4) has an unique almost periodic solution in $S$, which is uniformly asymptotically stable in $D$.

Theorem 4.3. Assume that (2.4) holds, and

$$
\begin{gathered}
b_{1}^{l}(t)+\frac{2 m_{2}^{\epsilon} m_{1}^{\epsilon}}{\left(m^{2}+\left(M_{1}^{\epsilon}\right)^{2}\right)^{2}}-\frac{M_{2}^{\epsilon}}{\left(m_{1}^{\epsilon}\right)^{2}}>0, \\
\frac{1}{M_{1}^{\epsilon}}-a_{1}^{u}(t)-\frac{1}{\left(m^{2}+\left(m_{1}^{\epsilon}\right)^{2}\right)}>0,
\end{gathered}
$$

where $M_{i}^{\epsilon}, m_{i}^{\epsilon}, i=1,2$, are defined in (2.6). Then system (1.6) has an unique positive almost periodic solution, which is globally asymptotically stable, especially uniformly globally asymptotically stable in $\Gamma_{\epsilon}^{*}$. 
Consider the product system of (4.2)

$$
\begin{gathered}
\frac{d \tilde{H}_{1}(t)}{d t}=r_{1}(t)-\frac{a_{1}(t)}{m^{2}+\exp \left(2 \tilde{H}_{1}(t)\right)} \exp \left(\tilde{P}_{1}(t)\right)-b_{1}(t) \exp \left(\tilde{H}_{1}(t)\right), \\
\frac{d \tilde{P}_{1}(t)}{d t}=r_{2}(t)-a_{2}(t) \frac{\exp \left\{\tilde{P}_{1}(t)\right\}}{\exp \left\{\tilde{H}_{1}(t)\right\}}, \\
\frac{d \tilde{H}_{2}(t)}{d t}=r_{1}(t)-\frac{a_{1}(t)}{m^{2}+\exp \left(2 \tilde{H}_{2}(t)\right)} \exp \left(\tilde{P}_{2}(t)\right)-b_{1}(t) \exp \left(\tilde{H}_{2}(t)\right), \\
\frac{d \tilde{P}_{2}(t)}{d t}=r_{2}(t)-a_{2}(t) \frac{\exp \left\{\tilde{P}_{2}(t)\right\}}{\exp \left\{\tilde{H}_{2}(t)\right\}} .
\end{gathered}
$$

Now we define a Lyapunov function on $[0,+\infty) \times \Gamma_{\epsilon}^{*} \times \Gamma_{\epsilon}^{*}$ as follows:

$$
V\left(t, \tilde{H}_{1}, \widetilde{P}_{1}, \tilde{H}_{2}, \widetilde{P}_{2}\right)=\left|\tilde{H}_{1}(t)-\tilde{H}_{2}(t)\right|+\left|\tilde{P}_{1}(t)-\widetilde{P}_{2}(t)\right|
$$

Then, condition (i) in Lemma 4.2 is satisfied for $\alpha(\gamma)=\beta(\gamma)=\gamma$ for $\gamma \geq 0$. In addition,

$$
\begin{aligned}
\mid V(t, & \left.\tilde{H}_{1}, \widetilde{P}_{1}, \tilde{H}_{2}, \widetilde{P}_{2}\right)-V\left(t, \tilde{H}_{3}, \widetilde{P}_{3}, \tilde{H}_{4}, \widetilde{P}_{4}\right) \mid \\
& =\left|\left(\left|\tilde{H}_{1}(t)-\tilde{H}_{2}(t)\right|+\left|\widetilde{P}_{1}(t)-\widetilde{P}_{2}(t)\right|\right)-\left(\left|\tilde{H}_{3}(t)-\widetilde{H}_{4}(t)\right|+\left|\widetilde{P}_{3}(t)-\widetilde{P}_{4}(t)\right|\right)\right| \\
& \leq\left|\tilde{H}_{1}(t)-\tilde{H}_{3}(t)\right|+\left|\widetilde{P}_{1}(t)-\widetilde{P}_{3}(t)\right|+\left|\tilde{H}_{2}(t)-\tilde{H}_{4}(t)\right|+\left|\widetilde{P}_{2}(t)-\widetilde{P}_{4}(t)\right| \\
& \leq||\left(\tilde{H}_{1}(t), \widetilde{P}_{1}(t)\right)-\left(\tilde{H}_{3}(t), \widetilde{P}_{3}(t)\right)||+||\left(\tilde{H}_{2}(t), \widetilde{P}_{2}(t)\right)-\left(\tilde{H}_{4}(t), \widetilde{P}_{4}(t)\right)||,
\end{aligned}
$$

which shows that the condition (ii) of Lemma 4.2 is also satisfied.

Let $\left(\tilde{H}_{i}(t), \widetilde{P}_{i}(t)\right), i=1,2$, be any two solutions of $(4.2)$ defined on $[0,+\infty) \times \Gamma_{\epsilon}^{*} \times \Gamma_{\epsilon}^{*}$.

Calculating the right derivative $D^{+} V(t)$ of $V(t)$ along the solutions of (4.2), we have

$$
\begin{aligned}
D^{+} V(t)= & \operatorname{sgn}\left(\tilde{H}_{1}(t)-\tilde{H}_{2}(t)\right)\left(-\frac{a_{1}(t)}{m^{2}+\exp \left(2 \tilde{H}_{1}(t)\right)}\left(\exp \left(\widetilde{P}_{1}(t)\right)-\exp \left(\widetilde{P}_{2}(t)\right)\right)\right. \\
& \left.-b_{1}(t)\left(\exp \left(\tilde{H}_{1}(t)\right)-\exp \left(\tilde{H}_{2}(t)\right)\right)\right) \\
& +\operatorname{sgn}\left(\widetilde{P}_{1}(t)-\widetilde{P}_{2}(t)\right)\left(-a_{2}(t)\left(\frac{\exp \left(\widetilde{P}_{1}(t)\right)}{\exp \left(\tilde{H}_{1}(t)\right)}-\frac{\exp \left(\widetilde{P}_{2}(t)\right)}{\exp \left(\tilde{H}_{2}(t)\right)}\right)\right)
\end{aligned}
$$




$$
\begin{aligned}
\leq & -\left(b_{1}^{l}+\frac{\exp \left(\tilde{P}_{2}(t)\right)\left(\exp \left(\tilde{H}_{1}(t)\right)+\exp \left(\tilde{H}_{2}(t)\right)\right)}{\left(m^{2}+\exp \left(2 \tilde{H}_{1}(t)\right)\right)\left(m^{2}+\exp \left(2 \tilde{H}_{2}(t)\right)\right)}-\frac{P(t)}{H(t) H^{*}(t)}\right. \\
& \left.-\frac{\exp \left(\widetilde{P}_{1}(t)\right)}{\exp \left(\tilde{H}_{1}(t)\right) \exp \left(\tilde{H}_{2}(t)\right)}\right) \times\left|\exp \left(\tilde{H}_{1}(t)\right)-\exp \left(\tilde{H}_{2}(t)\right)\right| \\
& -\left(\frac{1}{\exp \left(\tilde{H}_{2}(t)\right)}-a_{1}^{u}-\frac{1}{\left(m^{2}+\exp \left(2 \tilde{H}_{1}(t)\right)\right)}\right) \times\left|\exp \left(\widetilde{P}_{1}(t)\right)-\exp \left(\widetilde{P}_{2}(t)\right)\right| \\
\leq & -\left(b_{1}^{l}+\frac{2 m_{2}^{\epsilon} m_{1}^{\epsilon}}{\left(m^{2}+\left(M_{1}^{\epsilon}\right)^{2}\right)^{2}}-\frac{M_{2}^{\epsilon}}{\left(m_{1}^{\epsilon}\right)^{2}}\right)\left|\exp \left(\tilde{H}_{1}(t)\right)-\exp \left(\tilde{H}_{2}(t)\right)\right| \\
& -\left(\frac{1}{M_{1}^{\epsilon}}-a_{1}^{u}-\frac{1}{\left(m^{2}+\left(m_{1}^{\epsilon}\right)^{2}\right)}\right)\left|\exp \left(\widetilde{P}_{1}(t)\right)-\exp \left(\widetilde{P}_{2}(t)\right)\right| .
\end{aligned}
$$

Note that

$$
\begin{gathered}
\exp \left(\tilde{H}_{1}(t)\right)-\exp \left(\tilde{H}_{2}(t)\right)=\exp (\xi(t))\left(\tilde{H}_{1}(t)-\tilde{H}_{2}(t)\right), \\
\exp \left(\widetilde{P}_{1}(t)\right)-\exp \left(\widetilde{P}_{2}(t)\right)=\exp (\eta(t))\left(\widetilde{P}_{1}(t)-\widetilde{P}_{2}(t)\right),
\end{gathered}
$$

where $\xi(t)$ lies between $\tilde{H}_{1}(t)$ and $\tilde{H}_{2}(t)$ while $\eta(t)$ lies between $\widetilde{P}_{1}(t)$ and $\widetilde{P}_{2}(t)$. Then we have

$$
\begin{aligned}
D^{+} V(t) \leq & -\left(b_{1}(t)-\frac{M_{2}^{\epsilon}}{\left(m_{1}^{\epsilon}\right)^{2}}\right) m_{1}^{\epsilon}\left|\tilde{H}_{1}(t)-\tilde{H}_{2}(t)\right| \\
& -\left(\frac{1}{M_{1}^{\epsilon}}-a_{1}(t)\right) m_{2}^{\epsilon}\left|\widetilde{P}_{1}(t)-\widetilde{P}_{2}(t)\right| \\
\leq & -\mu\left(\left|\tilde{H}_{1}(t)-\widetilde{H}_{2}(t)\right|+\left|\widetilde{P}_{1}(t)-\widetilde{P}_{2}(t)\right|\right) \\
= & -\mu||\left(\tilde{H}_{1}(t), \widetilde{P}_{1}(t)\right)-\left(\tilde{H}_{2}(t), \widetilde{P}_{2}(t)\right) \|,
\end{aligned}
$$

where

$$
\mu=\min \left\{\left\{b_{1}^{l}+\frac{2 m_{2}^{\epsilon} m_{1}^{\epsilon}}{\left(m^{2}+\left(M_{1}^{\epsilon}\right)^{2}\right)^{2}}-\frac{M_{2}^{\epsilon}}{\left(m_{1}^{\epsilon}\right)^{2}}\right\},\left\{\frac{1}{M_{1}^{\epsilon}}-a_{1}^{u}-\frac{1}{\left(m^{2}+\left(m_{1}^{\epsilon}\right)^{2}\right)}\right\}\right\}>0 .
$$

Hence, the condition (iii) of Lemma 4.2 is verified as well. Therefore, by Theorem 4.1 and Lemma 4.2, it follows that system (4.2) has a unique almost periodic solution in $\Gamma_{\epsilon}^{*}$, say $\left(\tilde{H}^{*}(t), \widetilde{P}^{*}(t)\right)$, which is uniformly asymptotically stable in $\Gamma_{\epsilon}^{*}$. Hence, system $(4.2)$ has a 
unique positive almost periodic solution $\left(H^{*}(t), P^{*}(t)\right)$ in $\Gamma_{\epsilon}^{*}$, which is uniformly asymptotically stable in $\Gamma_{\epsilon}^{*}$. By Theorem 2.12 , we have that $\left(H^{*}(t), P^{*}(t)\right)$ is globally asymptotically stable. The proof is complete.

\section{Acknowledgments}

The first author was supported by the NSF of Gansu Province of China (3ZS042-B25013), the NSF of Bureau of Education of Gansu Province of China (0416B-08), the Key Research and Development Program for Outstanding Groups of Lanzhou University of Technology, and the Development Program for Outstanding Young Teachers in Lanzhou University of Technology. The second author was supported by the NNSF of China (10571078) and the Teaching and Research Award Program for Outstanding Young Teachers in Higher Education Institutions of Ministry of Education of China.

\section{References}

[1] J. F. Andrews, A mathematical model for the continuous culture of microorganisms utilizing inhibitory substrates, Biotechnology and Bioengineering 10 (1986), no. 6, 707-723.

[2] I. Barbalat, Systems d'equations differentielle d'oscillations nonlineaires, Revue Roumaine de Mathématiques Pures et Appliquées 4 (1959), 267-270.

[3] A. W. Bush and A. E. Cook, The effect of time delay and growth rate inhibition in the bacterial treatment of wastewater, Journal of Theoretical Biology 63 (1976), no. 2, 385-395.

[4] J. B. Collings, The effects of the functional response on the bifurcation behavior of a mite predatorprey interaction model, Journal of Mathematical Biology 36 (1997), no. 2, 149-168.

[5] Y.-H. Fan, W.-T. Li, and L.-L. Wang, Periodic solutions of delayed ratio-dependent predator-prey models with monotonic or nonmonotonic functional responses, Nonlinear Analysis. Real World Applications 5 (2004), no. 2, 247-263.

[6] M. Fan, Q. Wang, and X. Zou, Dynamics of a non-autonomous ratio-dependent predator-prey system, Proceedings of the Royal Society of Edinburgh. Section A. Mathematics 133 (2003), no. $1,97-118$.

[7] R. E. Gaines and J. L. Mawhin, Coincidence Degree, and Nonlinear Differential Equations, Springer, Berlin, 1977.

[8] K. Gopalsamy, Stability and Oscillations in Delay Differential Equations of Population Dynamics, Mathematics and Its Applications, vol. 74, Kluwer Academic, Dordrecht, 1992.

[9] H.-F. Huo and W.-T. Li, Existence and global stability of positive periodic solutions of a discrete delay competition system, International Journal of Mathematics and Mathematical Sciences 2003 (2003), no. 38, 2401-2413.

[10] , Periodic solutions of a periodic two-species competition model with delays, International Journal of Applied Mathematics 12 (2003), no. 1, 13-21.

[11]_, Periodic solution of a delayed predator-prey system with Michaelis-Menten type functional response, Journal of Computational and Applied Mathematics 166 (2004), no. 2, 453-463.

[12] _ Periodic solutions of a ratio-dependent food chain model with delays, Taiwanese Journal of Mathematics 8 (2004), no. 2, 211-222.

[13] - Positive periodic solutions of a class of delay differential system with feedback control, Applied Mathematics and Computation 148 (2004), no. 1, 35-46.

[14] H.-F. Huo, W.-T. Li, and R. P. Agarwal, Optimal harvesting and stability for two species stagestructured system with cannibalism, International Journal of Applied Mathematics 6 (2001), no. 1, 59-79. 
[15] H.-F. Huo, W.-T. Li, and S. S. Cheng, Periodic solutions of two-species diffusion models with continuous time delays, Demonstratio Mathematica 35 (2002), no. 2, 433-446.

[16] Y. Kuang, Delay Differential Equations with Applications in Population Dynamics, Mathematics in Science and Engineering, vol. 191, Academic Press, Massachusetts, 1993.

[17] P. H. Leslie, Some further notes on the use of matrices in population mathematics, Biometrika 35 (1948), no. 3-4, 213-245.

[18] A stochastic model for studying the properties of certain biological systems by numerical methods, Biometrika 45 (1958), no. 1-2, 16-31.

[19] Y. K. Li and Y. Kuang, Periodic solutions of periodic delay Lotka-Volterra equations and systems, Journal of Mathematical Analysis and Applications 255 (2001), no. 1, 260-280.

[20] E. C. Pielou, Mathematical Ecology, John Wiley \& Sons, New York, 1977.

[21] S. Ruan and D. Xiao, Global analysis in a predator-prey system with nonmonotonic functional response, SIAM Journal on Applied Mathematics 61 (2001), no. 4, 1445-1472.

[22] W. Sokol and J. A. Howell, Kinetics of phenol oxidation by washed cells, Biotechnology and Bioengineering 23 (1980), 2039-2049.

[23] Q. Wang, M. Fan, and K. Wang, Dynamics of a class of nonautonomous semi-ratio-dependent predator-prey systems with functional responses, Journal of Mathematical Analysis and Applications 278 (2003), no. 2, 443-471.

[24] L.-L. Wang and W.-T. Li, Existence of periodic solutions of a delayed predator-prey system with functional response, International Journal of Mathematical Sciences 1 (2002), no. 1-2, 55-63.

[25] __ Existence and global stability of positive periodic solutions of a predator-prey system with delays, Applied Mathematics and Computation 146 (2003), no. 1, 167-185.

[26] _ Periodic solutions and permanence for a delayed nonautonomous ratio-dependent predator-prey model with Holling type functional response, Journal of Computational and Applied Mathematics 162 (2004), no. 2, 341-357.

[27] D. Xiao and S. Ruan, Multiple bifurcations in a delayed predator-prey system with nonmonotonic functional response, Journal of Differential Equations 176 (2001), no. 2, 494-510.

[28] T. Yoshizawa, Stability Theory and the Existence of Periodic Solutions and Almost Periodic Solutions, Springer, New York, 1975.

Hai-Feng Huo: Department of Applied Mathematics, Lanzhou University of Technology, Lanzhou, Gansu 730050, China

E-mail address: hfhuo@lut.cn

Wan-Tong Li: School of Mathematics and Statistics, Lanzhou University, Lanzhou, Gansu 730000, China

E-mail address: wtli@lzu.edu.cn 


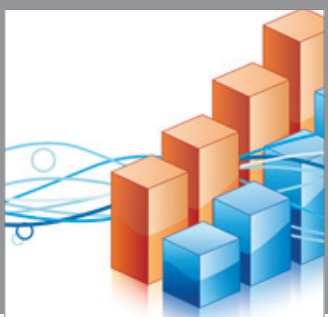

Advances in

Operations Research

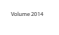

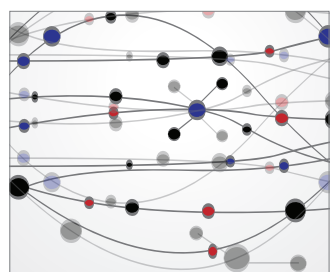

\section{The Scientific} World Journal
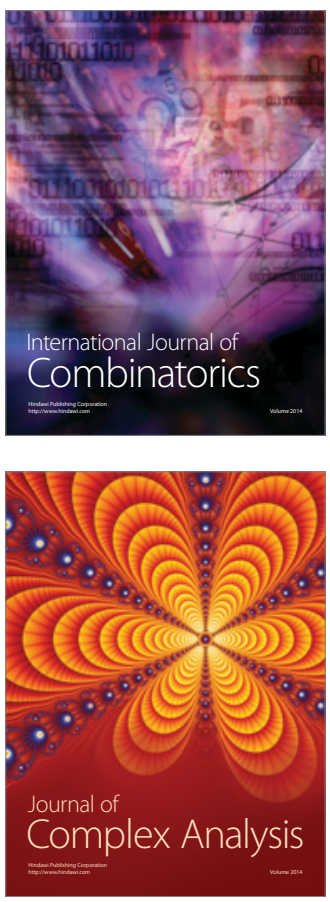

International Journal of

Mathematics and

Mathematical

Sciences
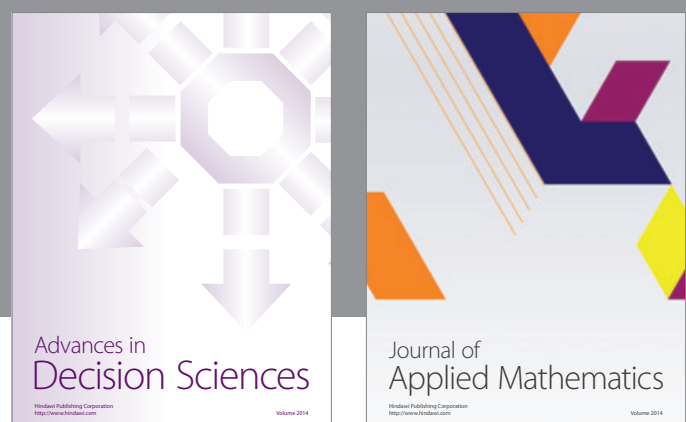

Journal of

Applied Mathematics
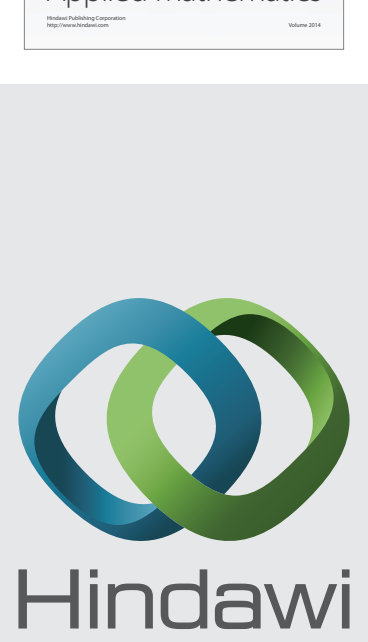

Submit your manuscripts at http://www.hindawi.com
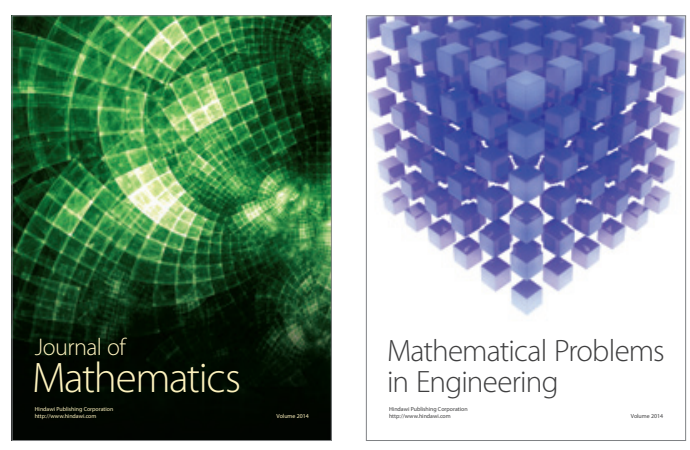

Mathematical Problems in Engineering
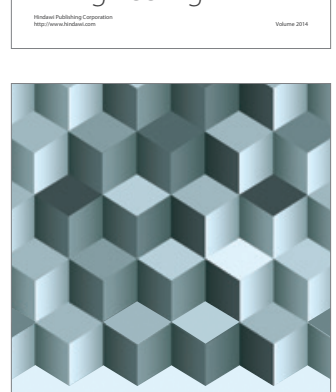

Journal of

Function Spaces
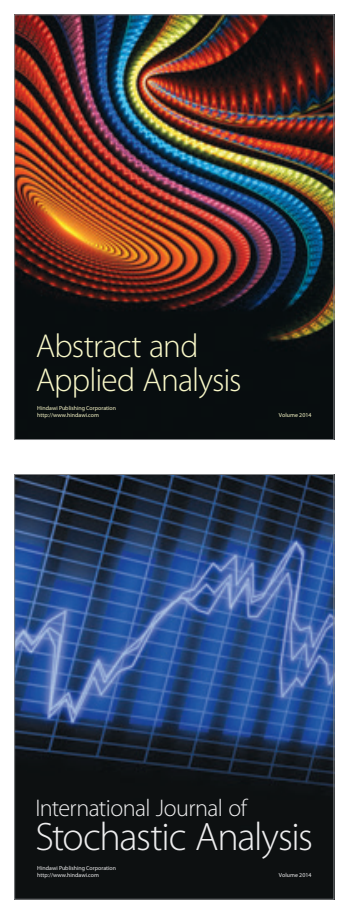

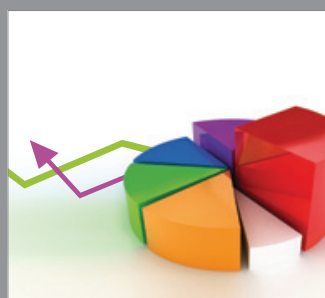

ournal of

Probability and Statistics

Promensencen
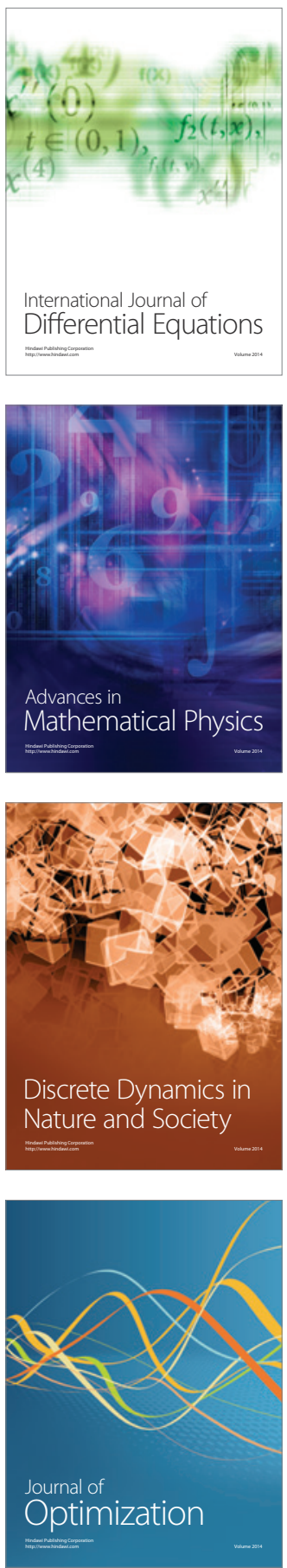